\title{
ASSOCIATIONS OF DUAL TASK EXERGAMING WITH COGNITIVE-MOTOR INTERFERENCE IN OLDER ADULTS WITH MILD COGNITIVE IMPAIRMENT: A SINGLE-ARM PILOT STUDY
}

\author{
L. Kannan ${ }^{1}$, T. Bhatt ${ }^{1}$
}

\begin{abstract}
Purpose: To examine the feasibility and effectiveness of dual task (DT) exergaming to improve volitional balance control in older adults with mild cognitive impairment (MCI). Methods: Ten older adults with MCI were examined at baseline (week-0) and post-training (week-5) on volitional balance control (maximum excursion of center of gravity, MXE [\%]) while performing cognitive task (auditory clock test or letter number sequencing task) and on the NIH-motor and cognitive toolboxes. DT exergaming training lasted for 12sessions which consisted of performing explicitcognitive tasks while playing the Wii-Fitbalance games. Results: From pre- to post-training, MXE improved ( $\mathrm{p}<0.05)$; however, cognitive accuracy (cognitive task) remained the same ( $\mathrm{p}>0.05$ ). Improvement in $\mathrm{NIH}$ motor and cognitive toolbox tests was observed post-training $(\mathrm{p}<0.05)$. Conclusion: DT exergaming was associated to improvements in balance control under attention-demanding conditions in MCI. Future studies may focus on examining the efficacy of such training.
\end{abstract}

Key words: Dual task training, exergaming, mild cognitive impairment, cognitive motor interference.

\section{Introduction}

Mild cognitive impairment (MCI), a prodromal stage of dementia, affecting about $15 \%$ to $20 \%$ of the older adults above the age of 65 in the United States (1) is characterized by substantial memory decline but preserved general intellectual function with subtle balance and gait deficits (2-4). Such deficits are more pronounced during dual tasking (simultaneous performance of cognitive and motor task) resulting in increased cognitive-motor interference (deteriorated performance on either one or both tasks) - a factor likely contributing to the higher fall risk in MCI (3, 5, 6). Therefore, studies have focused on dual task (DT) interventions to enhance and/or preserve the ability to allocate attentional resources to both balance and cognitive tasks when performed concurrently, often needed in daily living (ADLs) $(7,8)$.

Conventional DT training that involves repeated

1. Department of Physical Therapy, University of Illinois at Chicago. Chicago, USA

Corresponding Author: Tanvi Bhatt, PT, PhD, Department of Physical Therapy, 1919, W Taylor St, (M/C 898), University of Illinois at Chicago, Chicago, USA 60612, Email - tbhatt6@uic.edu, Phone - +1(312)-355-4443, Fax - +1(312)-996-4583

Received May 15, 2021

Accepted for publication September 1, 2021 practice of balance and/or gait activities simultaneously performed with cognitive tasks helps improve motor performance on ADLs but has limited benefits in improving cognitive performance $(7,8)$. Despite this, barriers such as lack of motivation, adherence to therapy, and limited access to rehabilitation facilities have led to development of alternate therapies involving exergamebased training (9-12). Such training provides real-time biofeedback (visual, auditory, tactile) on movement performance (delivered via low-cost commercial devices - Wii-fit) while implicitly facilitating cognitive domains and is known to be feasible, effective, and highly compliant among MCI (12).

It is known that MCI demonstrate significant structural and functional brain changes associated with executive dysfunction, deteriorated DT performance, and increased fall risk $(13,14)$. Additionally, exergame-based training may not implicitly address "executive function" (12, 15), and explicit cognitive training may aid in delaying or reversing the apparent cognitive decline. One study revealed promising effects on reducing cognitive-motor interference after 6 weeks of Wii-Fit + cognitive training (DT exergaming) in people with chronic stroke (16). As MCI also show significant cortical pathology, it could be postulated that a similar training may be similarly 
beneficial $(13,14)$.

Therefore, this single-arm pilot study aimed to examine the feasibility of a 4-week (12 sessions) DT exergaming intervention among MCI on self-initiated (volitional) balance control tasks under attentional demanding conditions (interference task). We hypothesized that MCI participants would show significant improvement in volitional balance, cognitive accuracy, and improved performance-based motor and cognitive function posttraining.

\section{Methods}

\section{Participants}

Older adults ( $>55$ years) were recruited from the University of Illinois Hospital Geriatric Clinic and flyers in nearby independent living senior centers and grocery stores. This study was approved by the University of Illinois at Chicago institutional review board. Ten older adults participated in the study after obtaining a written informed consent.

\section{Participants' eligibility}

To be included, participants must score 18-24 out of 30 on the Montreal Assessment on Cognitive Assessment (MoCA). Participants with uncontrolled cardiovascular disease, presence of any neurological condition (e.g., Alzheimer's disease), and/or severe musculoskeletal diseases that may interfere with the ability to receive the intervention were excluded. Additionally, people with the inability to stand independently without an assistive device for the length of a Wii-Fit game, with a fracture risk heel bone density (measured using Lunar Achilles Insight) $\mathrm{T}$-score $<-2.0$, and inability to communicate and understand English were excluded.

\section{Research Design}

This was a single arm pre-post research design consisting of 4 weeks of DT exergaming sessions. Baseline (at week 0) and post-testing (at 5th week) outcome measures were collected.

\section{Intervention}

In total, 12 sessions of individual one-on-one DT exergaming was administered and supervised by a research personnel (physical therapist) in a research facility. Participants wore a gait belt and were supervised during the session. DT exergaming was delivered via Wii-Fit standing balance games which was performed at light intensity (rate of perceived exertion via Borg's scale with individuals reporting score of 7-11) (17) and was concurrently performed with explicit cognitive games (therapist cued) for 90 minutes/session, 3 times / week. While Wii fit games implicitly addressed cognitive domains like working memory, episodic memory, and visuospatial awareness, explicit cognitive games targeted subdomains of executive function - working memory and attention, and semantic memory, abstract memory. Warm up (step-in-place, trunk twists) and cool down (stretching of lower limb) were performed before and after session, respectively. Refer to supplementary material section for details of protocol.

\section{Assessments}

Volitional balance control task: The limits of stability (LOS) test via Balance Master (Equitest ${ }^{\circledR}$ Neurocom) (18) was administered. Participants were secured in a safety harness and asked to stand on the force platform of the Balance Master (Figure 1). Participants were instructed to lean their body either in the forward, backward, left, or right direction to move their center of gravity (COG) projection shown on a screen to the desired direction without losing balance, stepping, or reaching for assistance.

\section{Figure 1}

Represents a picture of an individual performing the limits of stability test on Balance Master (Equitest ${ }^{\circledR}$

Neurocom) in the forward direction
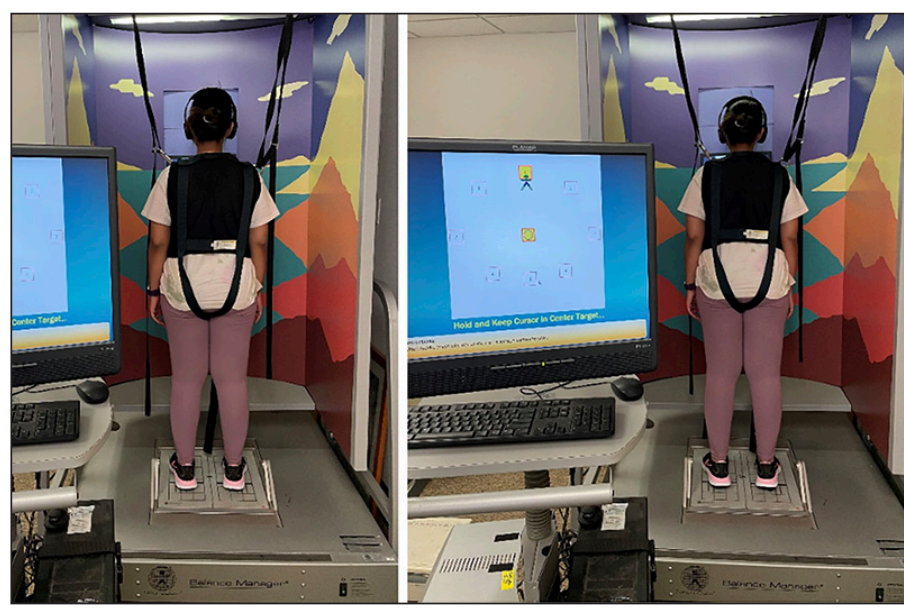

\section{Cognitive task}

Auditory clock test (ACT) (19) and letter number sequencing task (LNS) (20) were administered using the DirectRT EmpirisoftTM (21) software to assess subdomains of executive function (visuo-spatial memory, working memory, attention, and cognitive flexibility). The audio cues were delivered through headphones and responses were recorded through a microphone. The ACT involved responding to different times of the day, "yes" if the hour and the minute hand was on the same side of the clock face and "no" otherwise. The LNS 
involved sequentially listing alternate letter and number combinations, for example, response to "C5," was D6, E7, etc.

\section{Interference test}

The LOS test (all directions) was performed along with both cognitive tasks mentioned above. Participants began responding to the cognitive cues followed by the LOS task.

\section{NIH Toolbox}

An iPad was used to test the motor and cognitive domains. The motor tests include 4-meter gait speed test and 2-minute walk test. The cognitive tests include list sort memory test (working memory), picture sequence memory test (episodic memory), dimensional change card sort test (executive function), flanker inhibitory control and attention test (attention and executive function), and pattern comparison processing speed test (processing speed) (refer supplementary material section).

\section{Outcome measures}

Volitional balance control and interference task: Single task (task when performed alone) and performance during interference task was quantified by the movement stability measurement of maximum excursion (MXE, expressed in percentage), which is the maximum ability to shift one's COG toward the theoretical limit in the desired direction. Higher values indicate better performance.

Cognitive and interference task: Accuracy [(Correct responses)(Total responses) ${ }^{*} 100$ ] was calculated during single and interference task.

$\mathrm{NIH}$ toolbox: Speed $(\mathrm{m} / \mathrm{sec})$ for 4 -meter gait test and distance covered in 2-minute walk test for endurance was computed. Number of correct responses for list sort memory test and accuracy for the remainder tests was included for analysis.

\section{Statistical analyses}

Statistical analyses were performed using SPSS version 24, Chicago, IL, USA. For MXE in each volitional balance control task direction (i.e., forward, backward, left, and right), $2 \times 2$ repeated measures analysis of variance (ANOVA) was performed to examine the time (pre- to post-training) and task (single vs. interference task) differences on with follow-up post-hoc tests. Similarly, four repeated measures ANOVA for accuracy (cognitive) in ACT and LNS was performed. Paired t-test was conducted for NIH toolbox measures. Refer supplementary material section for details.

\section{Results}

Demographics: Demographic characteristics of participants who completed the study are provided in Table 1.

Table 1

Demographics and clinical characteristics of older adults with mild cognitive impairment (MCI). BBS = Berg Balance Scale, MoCA = Montreal Cognitive

Assessment

\begin{tabular}{lc}
\hline & MCI \\
\hline Age [Means (SD)] & $64.1(5.74)$ \\
Range in years & $57-74$ \\
Gender (M/F) & $6 / 4$ \\
Dominance (R/L) & $10 / 0$ \\
Height (cm) [Means (SD)] & $171.5(9.63)$ \\
Range in cm & $152.4-181.61$ \\
Weight (lbs) [Means (SD)] & $167.14(35)$ \\
Range in lbs & $116-211$ \\
BBS Out of 56 [Means (SD)] Pre & $54.3(2.2)$ \\
Range & $49-56$ \\
BBS Out of 56 [Means (SD)] Post & $55.6(0.96)$ \\
Range & $53-56$ \\
MoCA Out of 30 [Means (SD)] & $21.4(1.42)$ \\
Range & $19-23$ \\
\hline
\end{tabular}

Volitional balance control and interference task: From pre- to post-training, MXE improved in the forward and left direction $(p<0.05)$ under interference test (Figure $2 a-2 d)$. Results of ANOVA and follow-up test are presented in Table 2.

Cognitive and interference task: Accuracy on ACT (Figure 2e-2h) and LNS (Figure 1i-1l) showed significant improvement only during single task performances $(\mathrm{p}<0.05)$, however, no improvement was observed during interference test $(p>0.05)$. Results of ANOVA and followup test are presented in Table 3.

NIH toolbox: A significant increase in gait speed was observed post-training $(\mathrm{p}<0.05)$; however, there was no change in the 2-minute walk test distance covered ( $p>0.05)$ (Figure 3a). Post-training, significant improvements in NIH cognitive toolbox measures of working memory $(p<0.05)$ (Figure $3 b)$, episodic memory $(p<0.01)$ (Figure $3 c)$, and executive function $(p<0.01)$ (Figure 3d) were observed. However, no improvements were observed in attention $(p>0.05)$ and processing speed $(p>0.05)$. 
Table 2

Results for balance control task

\begin{tabular}{|c|c|c|c|c|c|c|}
\hline \multicolumn{7}{|c|}{$3 \times 2$ repeated measure ANOVA } \\
\hline $\begin{array}{l}\text { Main effects and inte- } \\
\text { raction }\end{array}$ & $\begin{array}{c}\text { Df } \\
\text { (df1,df2) }\end{array}$ & & $\begin{array}{l}\text { Forward lean } \\
\text { F Values }\end{array}$ & $\begin{array}{l}\text { Backward lean } \\
\text { F Values }\end{array}$ & $\begin{array}{c}\text { Left } \\
\text { lean } \\
\text { F Values }\end{array}$ & $\begin{array}{c}\text { Right } \\
\text { lean } \\
\text { F Values }\end{array}$ \\
\hline Task effect & 2,36 & & $6.987^{* *}$ & 2.389 & $8.807^{* * *}$ & 1.826 \\
\hline Time effect & 1,18 & & 2.733 & 2.277 & 3.841 & 2.729 \\
\hline Group $\times$ Time & 2,36 & & 2.349 & 0.382 & $4.069^{*}$ & 1.496 \\
\hline \multicolumn{4}{|c|}{ Paired t-test } & \multicolumn{3}{|c|}{ Pre vs Post training } \\
\hline Balance Tasks & & Df & & ST & ACT & LNS \\
\hline Forward & & 1,9 & & -2.148 & $-4.117^{* *}$ & $-2.605^{*}$ \\
\hline Backward & & 1,9 & & -1.539 & -0.229 & -1.783 \\
\hline Left sideways lean & & 1,9 & & -1.08 & $-2.707^{*}$ & $-2.438^{*}$ \\
\hline Right sideways lean & & 1,9 & & $-2.336^{*}$ & -1.729 & -0.736 \\
\hline
\end{tabular}

ST: single task ; ACT: Auditory clock test; LNS: Letter number sequencing; ${ }^{*} \mathrm{p}<0.05^{* *} \mathrm{p}<0.01^{* * *} \mathrm{p}<0.001$

Table 3

Results for cognitive task

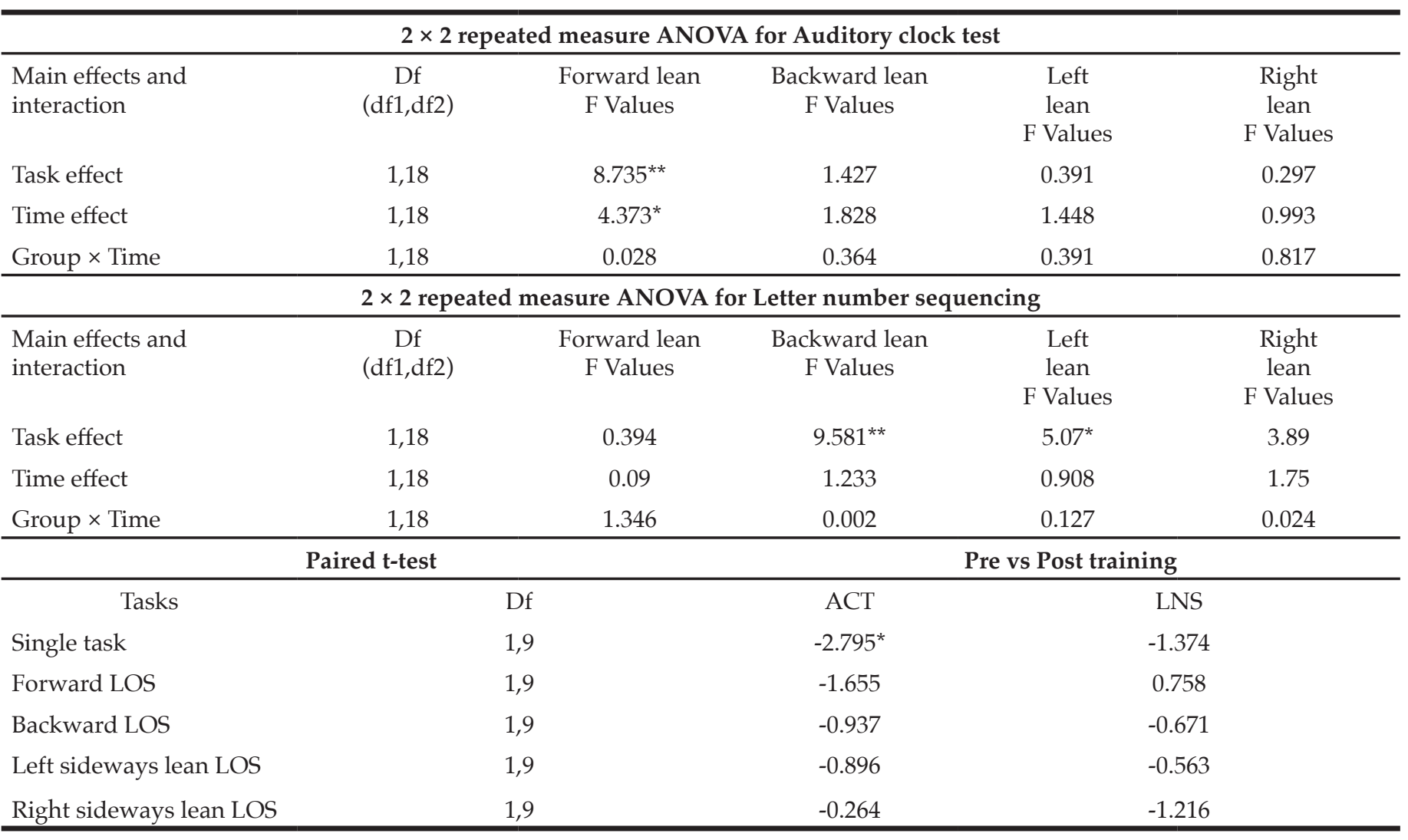

ST: single task; ACT: Auditory clock test; LNS: Letter number sequencing; ${ }^{*} \mathrm{p}<0.05{ }^{* *} \mathrm{p}<0.01{ }^{* * *} \mathrm{p}<0.001$ 
Figure 2

Association of dual task training with balance control and cognition under single and dual task conditions

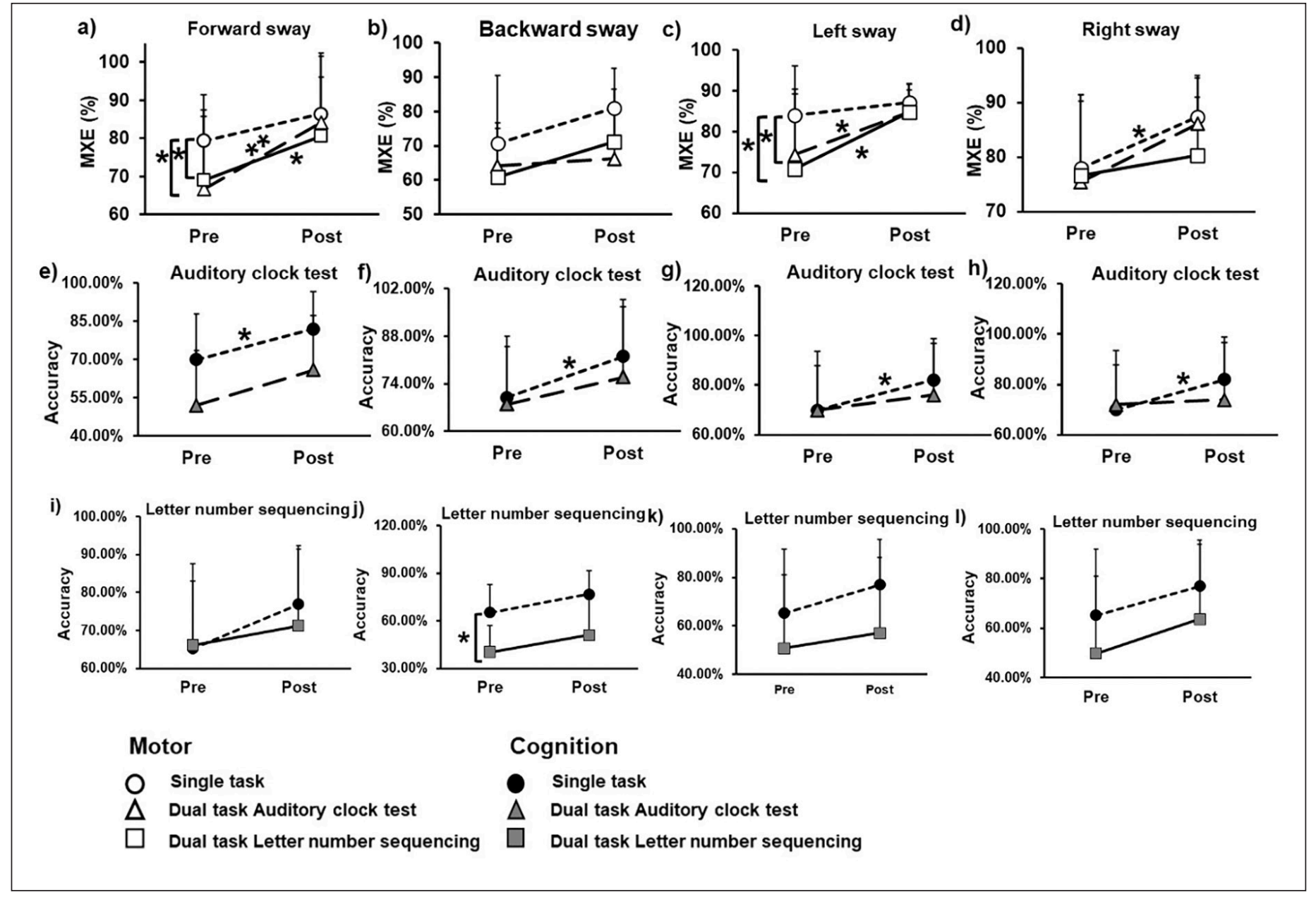

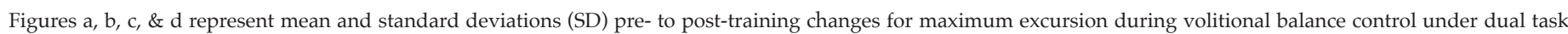

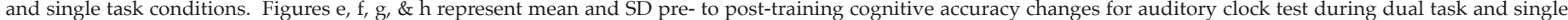

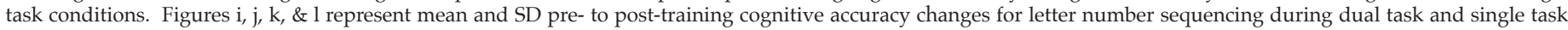
conditions

\section{Discussion}

As hypothesized, the study results showed a significant improvement in volitional balance control under interference conditions but no improvement in ACT and LNS. Additionally, the intervention resulted in improved gait speed but not endurance for motor function and, similarly, improved working memory, episodic memory, and executive function but not attention and processing speed.

With respect to interference task conditions, the results show improvement in motor performance but no change in ACT and LNS cognitive tasks demonstrating motor prioritization (6). Repeated practice of multidirectional weight shift training that challenged one's LOS and immediate biofeedback (visual) with knowledge of performance during training could have facilitated the ability to precisely control one's center of mass (COM) body movement. The LOS test utilizes a significant amount of attentional resources within the dorsolateral prefrontal cortex (DLPFC, associated with executive functions) (22). Post-training, utilization of the shared resources between cognitive and motor areas perhaps improved by channeling available attentional resources to prioritize motor performance - probably due to the $\mathrm{CNS}^{\prime}$ estimation or perception of the balance task to be more challenging with significant consequences (such as falls) in case of failure. Furthermore, the significantly greater pathology affecting the DLPFC and associative sensorimotor areas (controlling executing function) than the premotor or motor areas (controlling volitional balance) could attribute to motor prioritization (5).

Our study yielded motor benefits in forward and left lean but did not improve backward and right lean. It has been postulated that backward leans are more difficult than forward leans due to directionalspecific anatomic constraints and increased reliance on proprioceptive and vestibular systems (over visual system) in older adults (23-25). Aging-induced changes causes impaired integration of these systems and any 
Figure 3

Means and SD for gait speed shown in meters $(\mathrm{m})$ and obtained from the NIH motor toolbox; and b) working memory tested via list sort memory test, (c) episodic memory tested via picture sequence memory test, and (d) executive function via dimensional change card sort obtained from the NIH cognitive toolbox

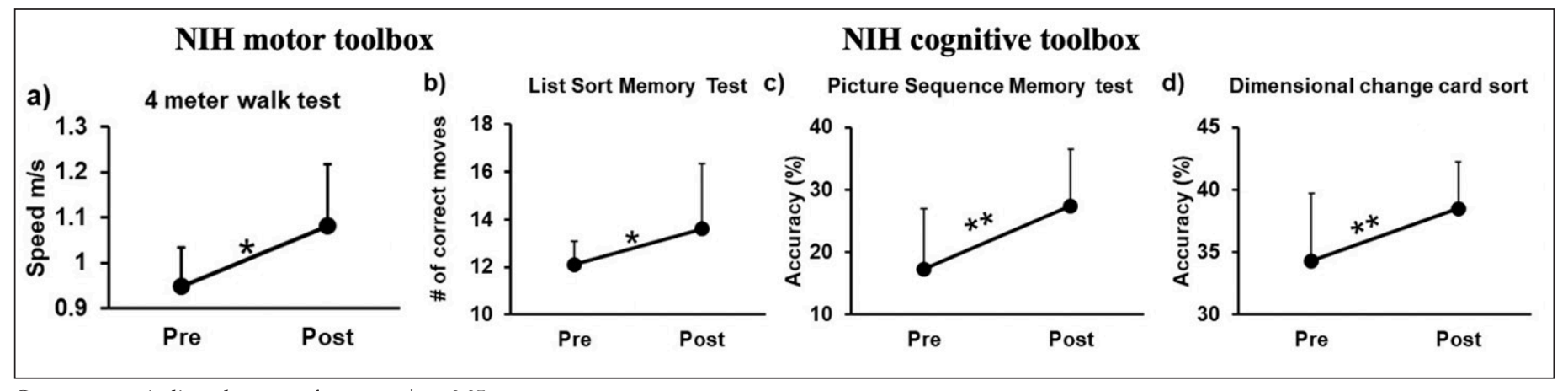

Greater scores indicate better performance. ${ }^{*}, \mathrm{p}<0.05$

cognitive decline further depreciates this sensory signal processing, resulting in deteriorated balance control (23, 24). Thus, impaired cognitive-sensory signal processing could explain the poor performance and lowest MXE for backward lean at baseline (single and interference task). Lastly, motor performance was the highest on right lean, which could be related to the dominance/preferred side for performing activities and, therefore, there may have been limited room for improvement in the right lean.

Although there wasn't an improvement in cognitive performance during interference task, visuo-spatial and working memory (ACT) improved under single task conditions. Apart from the implicit benefits of exergaming $(12,15)$, and the explicit cognitive training that targeted subdomains of executive function (attention, planning, and working memory) may explain such improvements. However, there were no benefits in cognitive flexibility (i.e., letter number sequencing) despite a positive trend. This task requires simultaneous utilization of attention, processing information, and working memory. Although the training did target these domains, the dosage might not be enough to induce change.

Similar to our study, studies targeting balance training in standing have shown improvements in overground gait speed in MCI (3) and positive transfer to improved mobility (16). This could be attributed to task-specific characteristics of exergames, which involve time limited stepping activities. However, our results showed no improvement in endurance. This could be because the exergames did not incorporate high-intensity training that is known to induce improvement in cardiovascular function in MCI $(7,8)$. Lastly, due to the implicit (via Wii fit games) and explicit (therapist cued cognitive tasks) cognitive training components of the protocol, improvement in NIH cognitive toolbox measures for executive function, attention, and processing speed were expected and agree with results of previous DT training studies $(7,12)$.
Despite the positive results, our study has certain limitations. Firstly, there was a small sample size and lack of a control group due to the study's preliminary nature. Furthermore, the training was limited to 4 weeks, and greater training dosage may yield larger motor and cognitive improvements under DT conditions. While our results demonstrate partial benefits of DT exergaming on balance control (self-initiated), its effects on the primary defense mechanism - reactive balance control - remain to be explored.

\section{Conclusion}

Our preliminary study demonstrated that DT exergaming has the potential to improve balance control and, limited benefits in executive function which could potentially have an impact in fall-risk reduction.

Conflict of interest: We have no conflict of interest to declare.

Ethical standards: The study was supported by Midwest Roybal Center for Health Promotion and Translation awarded to Dr. Tanvi Bhatt. This work was carried out after securing approval from the University of Illinois institutional review board protocol 2018-1257 and was registered on clinicaltrials.gov NCT03765398.

\section{References}

1. Langa, K.M. and D.A. Levine, The diagnosis and management of mild cognitive impairment: a clinical review. Jama, 2014. 312(23): p. 2551-2561.

2. Sheridan, P.L. and J.M. Hausdorff, The role of higher-level cognitive function in gait: executive dysfunction contributes to fall risk in Alzheimer's disease. Dementia and geriatric cognitive disorders, 2007. 24(2): p. 125-137.

3. Bahureksa, L., et al., The impact of mild cognitive impairment on gait and balance: a systematic review and meta-analysis of studies using instrumented assessment. Gerontology, 2017. 63(1): p. 67-83.

4. Lopez, O.L., et al., Neuropsychological characteristics of mild cognitive impairment subgroups. Journal of Neurology, Neurosurgery \& Psychiatry, 2006. 77(2): p. 159-165.

5. Muir, S.W., K. Gopaul, and M.M. Montero Odasso, The role of cognitive impairment in fall risk among older adults: a systematic review and metaanalysis. Age and ageing, 2012. 41(3): p. 299-308.

6. Plummer, P., et al., Cognitive-motor interference during functional mobility after stroke: state of the science and implications for future research. Archives of physical medicine and rehabilitation, 2013. 94(12): p. 2565-2574. e6.

7. Law, L.L., et al., Effects of combined cognitive and exercise interventions on cognition in older adults with and without cognitive impairment: a 
systematic review. Ageing research reviews, 2014. 15: p. 61-75.

8. Zhang, W., et al., Interventions to improve gait in older adults with cognitive impairment: a systematic review. Journal of the American Geriatrics Society, 2019. 67(2): p. 381-391.

9. Ben-Sadoun, G., et al., Physical and cognitive stimulation using an exergame in subjects with normal aging, mild and moderate cognitive impairment. Journal of Alzheimer's Disease, 2016. 53(4): p. 1299-1314.

10. Colombo, M., et al. Virtual reality for persons with dementia: an exergaming experience. in ISARC. Proceedings of the International Symposium on Automation and Robotics in Construction. 2012. IAARC Publications.

11. Ge, S., et al., Technology-based cognitive training and rehabilitation interventions for individuals with mild cognitive impairment: a systematic review. BMC geriatrics, 2018. 18(1): p. 1-19.

12. Zhao, Y., et al., Effectiveness of exergaming in improving cognitive and physical function in people with mild cognitive impairment or dementia: systematic review. JMIR serious games, 2020. 8(2): p. e16841.

13. Crockett, R.A., et al., Resting state default mode network connectivity, dual task performance, gait speed, and postural sway in older adults with mild cognitive impairment. Frontiers in aging neuroscience, 2017. 9: p. 423.

14. Zhang, Y., et al., MRI markers for mild cognitive impairment: comparisons between white matter integrity and gray matter volume measurements. PloS one, 2013. 8(6): p. e66367.

15. Stojan, R. and C. Voelcker-Rehage, A systematic review on the cognitive benefits and neurophysiological correlates of exergaming in healthy older adults. Journal of clinical medicine, 2019. 8(5): p. 734.

16. Kannan, L., et al., Cognitive-motor exergaming for reducing fall risk in people with chronic stroke: A randomized controlled trial. NeuroRehabilitation, 2019. 44(4): p. 493-510.

17. Worley, J.R., S.N. Rogers, and R.R. Kraemer, Metabolic responses to Wii Fit ${ }^{\mathrm{TM}}$ video games at different game levels. The Journal of Strength \& Conditioning Research, 2011. 25(3): p. 689-693.

18. Jbabdi, M., P. Boissy, and M. Hamel, Assessing control of postural stability in community-living older adults using performance-based limits of stability. BMC geriatrics, 2008. 8(1): p. 1-8.

19. Haggard, P. and J. Cockburn, Dividing attention between cognitive and motor tasks in neurological rehabilitation. Neuropsychological Rehabilitation, 1998. 8: p. 155-170.

20. Mrazik, M., S. Millis, and D.L. Drane, The oral trail making test: effects of age and concurrent validity. Archives of Clinical Neuropsychology, 2010. 25(3): p. 236-243.

21. Jarvis, W., MediaLab (Version 200x. xx)[Computer Software]. Empirisoft Corporation, 2017.

22. Nachev, P., C. Kennard, and M. Husain, Functional role of the supplementary and pre-supplementary motor areas. Nature Reviews Neuroscience, 2008 9(11): p. 856-869.

23. Berchicci, M., et al., Stepping forward, stepping backward: a movementrelated cortical potential study unveils distinctive brain activities. Behavioural brain research, 2020. 388: p. 112663.

24. Carbonneau, E. and C. Smeesters, Effects of age and lean direction on the threshold of single-step balance recovery in younger, middle-aged and older adults. Gait \& posture, 2014. 39(1): p. 365-371.

25. Duarte, M. and V.M. Zatsiorsky, Effects of body lean and visual information on the equilibrium maintenance during stance. Experimental brain research 2002. 146(1): p. 60-69. 